\title{
Corporeidad y aprendizaje en el contexto de la Enseñanza General Básica: comprensión y descripción de los procesos de construcción de conocimiento desde la acción relacional de los actores
}

\author{
Body movement and learning in primary school teaching: understanding and description \\ of knowledge building processes from the relational action of the actors \\ Corporeidade e aprendizagem no contexto do Ensino Básico Geral: compreensão e \\ descrição dos processos de construção do conhecimento a partir da ação relacional \\ dos atores
}

\author{
Sergio Toro A. ${ }^{\mathrm{a}}$ y Angela Niebles ${ }^{\mathrm{a}}$ \\ aUniversidad Austral de Chile. Correo electrónico: seatoro@gmail.com
}

\begin{abstract}
RESUMEN
La presente investigación se enmarca en un proceso de tres años, acerca de la relación entre la corporeidad y el aprendizaje en el contexto escolar, específicamente en el ciclo básico, desde diferentes áreas disciplinares o subsectores de aprendizaje. La base epistemológica se orienta desde la fenomenología (Noe, 2010; Merleau-Ponty, 2000) y las neurociencias (Varela, 2000; Thompson, 2007; Gallagher y Schmicking, 2010). El objetivo central se ubica en la caracterización y categorización de los despliegues corpóreos en función del aprendizaje autentico de los/as niños/as. La metodología utilizada es fundamentalmente cualitativa, específicamente estudio de casos, aunque se usaron ciertos análisis de cluster en función de caracterizar tipos de establecimientos escolares de enseñanza básica a estudiar. Los resultados fundamentales se orientan hacia procesos de acción y despliegue corpóreo fundamentalmente de dependencia y heteronomía, en las sesiones formales de clases, a diferencia de lo que ocurre en espacios de recreo y de libre acción. Tal situación permite interpretar que la escuela es fundamentalmente, en los casos estudiados, un lugar de relaciones de resistencia y domesticación, mas que de autonomía y construcción de las propias acciones.
\end{abstract}

Palabras clave: aprendizaje situado, corporeidad, acción, relacionalidad.

\begin{abstract}
This investigation was produced within a three years process, related to body experience and learning in school context, specifically in primary school, from different areas or teaching topics. The epistemological foundation is taken from the phenomenology (Noe, 2010; Merleau-Ponty, 2000) and the neurosciences (Varela, 2000; Thompson, 2007; Gallagher y Schmicking, 2010). The main objective is set on the characterization and categorization of body movement on the basis of learning in children. The methodology used is mainly qualitative, precisely case study, although some cluster analyses were used in order to categorize the schools under research. The main results are oriented to action and movement processes mainly in dependency and heteronomy in formal classes more than during school breaks or other spaces. This situation allows considering the school is essentially, in the analyzed cases, a space of resistance relationships and domestication rather than a place of autonomy and own actions development.
\end{abstract}

Key words: situated learning, body movement, action, relationship.

Investigación financiada por Fondecyt N N 11080297 “Corporeidad y aprendizaje, en el contexto de la enseñanza general básica: compresión y descripción de los procesos de construcción de conocimiento desde la acción relacional de los actores. (2008-2011), Fondecyt de Iniciación, Investigador Responsable. 


\section{RESUMO}

A pesquisa é parte de um processo de três anos sobre a relação entre corporeidade e aprendizagem no contexto escolar, especificamente no Ciclo Básico do Ensino Fundamental, a partir de diferentes áreas de aprendizagem. A base epistemológica é orientada pela fenomenologia (Noe, 2010; Merleau-Ponty, 2000) e neurociências (Varela, 2000; Thompson, 2007; Gallagher e Schmicking, 2010). Objetiva-se principalmente caracterizar e categorizar movimentos corpóreos em decorrência da aprendizagem autêntica das crianças. Fundamentalmente, utilizou-se metodologia qualitativa, especificamente o estudo de caso, embora a análise de cluster tenha sido utilizada em certos casos para caracterizar os tipos de instituições escolares de Ensino Básico a estudar. Resultados fundamentais se orientam para processos de ação e movimento corpóreo fundamentalmente de dependência e heteronomia, no tempo das aulas, diferentemente do que ocorre nos espaços de recreio e de atividade livre. Tal situação permite interpretar que a escola é fundamentalmente, nos casos estudados, um lugar de relações de resistência e domesticação, mais que de autonomia e construção de ações próprias.

Palavras chave: aprendizagem situada, movimento corporal, ação, relacionalidade.

\section{PRESENTACIÓN}

Desde la neurociencias, específicamente desde la visión de Francisco Varela (1997, 2000) definida como neurofenomenología, se ha establecido con muchas evidencias la estrecha relación que se genera entre la corporeidad, o la experiencia de ser cuerpo, y los procesos de aprendizaje. En tal sentido, este ultimo se puede comprender como un sinónimo de conocer, ${ }^{1}$ que permite en el transcurso de la vida social, la construcción de la conciencia, la individualidad y los procesos de distinción. Dicha relación se establece en los despliegues cotidianos de los sujetos en sus más diversos campos de relación y expresión, marcando y definiendo los contenidos y, por sobre todo, los procesos de aprendizaje o, dicho en otras palabras, las formas de aprender. Por lo tanto, dicho fenómeno descansa en una determinada forma de posicionarse y vincularse desde la experiencia y vivencia de un ser encarnado y de su acoplamiento con el entorno. La tesis fundamental de dicha opción se centra en plantear que la percepción es, en definitiva, una acción que se construye en la cotidianidad del vivir a través de procesos que no siempre son pre-meditados y definidos sistemáticamente desde la instrucción deliberada.

Este aspecto adquiere vital importancia si se considera la edad o etapa infantil en relación a la capacidad y potencialidad de aprendizaje y, por otra parte, a la escuela como institución responsable de desarrollar los aprendizajes socialmente instituidos como validos dentro de un determinado ordenamiento social. De ahí la pertinencia en preguntarse ¿cómo se considera desde la institucionalidad el aprendizaje como un proceso encarnado?; desde las dinámicas relacionales, ¿qué tipo de aprendizaje se experimenta, desarrolla y potencia? ¿Cómo se configura la especialidad y temporalidad en los procesos de aprendizaje de los niños y niñas en la enseñanza básica? En este sentido el objetivo general de la investigación es comprender los procesos de aprendizajes en el contexto del nivel escolar básico, desde las dinámicas personales y relacionales situados en la cotidianidad, como así también, desde la especialidad comprometida. Consecuentemente, se pretende describir los aprendizajes desarrollados desde la dimensión corpórea, dado que define lo que en definitiva los niños y niñas aprenden. Otro aspecto a destacar dentro de los objetivos específicos de la

\footnotetext{
1 En esta investigación se asume la propuesta de Maturana y Varela :"Todo hacer es un conocer y todo conocer es un hacer" (1990: 13), por lo tanto, el aprendizaje se constituye como un hacer definido y configurado desde el despliegue y repliegue de la corporeidad en su constante confluencia con el entorno.
} 
investigación es clasificar desde una matriz compresiva las dinámicas relacionales que se manifiestan en la escuela entre los más diversos actores y condiciones presentes en ella, entiéndase alumnos/as, docentes, espacialidad y organización escolar.

La opción para abordar la problemática dada las características de la misma es de complementariedad metodológica, vale decir, se asume la combinación de técnicas en primera, segunda y tercera persona (cuantitativos y cualitativos, en el primer caso, análisis de Cluster y un análisis interaccional en el segundo) a fin de establecer una comprensión del problema desde una matriz compleja del mismo (Morin, 1990). Se logra así validez y consistencia de los datos y hallazgos de la investigación. Dicha opción metodológica se implementa a partir de un trabajo de campo que contemple las diversas realidades socio-culturales del sistema escolar en la enseñanza escolar básica, municipal, particular subvencionado y particular pagado, a fin de discriminar el impacto sociocultural en los aprendizajes y en las diversas categorías expresadas en los objetivos de la investigación.

\section{FUNDAMENTOS EPISTEMOLÓGICOS}

En la actualidad, diferentes investigaciones emanadas de diferentes ciencias han desarrollado planteamientos sobre la posibilidad y condición del aprender y conocer del ser humano. En tal sentido, los aportes de la neurociencia (Varela y Thompson, 2001; Llinas, 2004; Damasio, 2000: Kandel et al 1997; Cosmelli, 2004; Maturana, 2004), de la filosofía (Lakoff y Johnson 1999; Zubiri, 1986; Morin, 2003; Castoriadis, 1997; Noe, 2004, 2009), la Inteligencia Artificial (Pfeifer y Bongard, 2007) y de la sicología (Valsiner, 2003; Pozo, 2001) nos permiten sintetizar la evidencia de la encarnación de la mente. Vale decir, en el caso de lo humano hablar de mente y de cuerpo es prácticamente un isomorfismo en términos experienciales.

Tal visión se ha desarrollado como una consecuencia de los primeros planteamientos de la fenomenología a principios del siglo XX con dos de sus más grandes autores, a saber, Husserl (1949) y Merleau-Ponty (2000). A partir de ellos, se comienza a desarrollar la idea de la conjunción de la mente y la carne, el conocido entre deux. Vale decir, un camino intermedio que nos permitiera establecer la unidad entre la experiencia y la idea, entre percepción y efección. Desde estos autores se establece lo que hoy señalamos como una cognición encarnada o mente encarnada.

En el logro de tal empresa se hace necesario revisar algunos de los supuestos planteados desde la fenomenología. El primer aspecto se relaciona con el sentir o la dimensión sensoria o explorativa (Toro, 2005) de la experiencia humana, vale decir, en cada acción que se emprende se explora y se conoce el mundo independiente del sentido o direccionalidad de la misma acción. En cada instante del proceso de ejecución de la misma, requiere una contrastación de la efectividad y de la amplitud, ritmo e intensidad de lo que se está ejecutando. Incluso si observamos la generación de la acción desde un punto de vista proyectivo, vale decir en una suerte de etapa de planificación u organización, las informaciones requeridas para esta fase requieren de algún tipo de afección o sentir del sí mismo y del entorno inmediato.

Ahora bien, podemos preguntarnos si la tematización del sentir, que procede de este modo regresivo, permite responder de vuelta a la ambición que anima el termino o signo, o sea, fundar la posibilidad de la palabra como tal. ¿Cómo establecer una continuidad entre el 
ser-ahí de la cosa, que manifiesta cierta positividad, y la evanescencia del sentido lingüísti$\operatorname{co}$ ¿Cómo pensar la diferencia de lo invisible enfrente a lo visible, diferencia que sostiene la posibilidad de la expresión, sin reducirlo a una entidad positiva? ¿En qué es "el mismo ser que percibe y que habla", y cuál es el ser de éste? La posibilidad de fundar la expresión lingüística, resultado último del aprendizaje, en el silencio de la percepción, es decir, pasar de un modo de proceder regresivo a un modo progresivo, requiere una reflexión aún más radical, orientada sobre la esencia misma del sentir. Podemos hoy día señalar que es en la motricidad que consiste esta esencia, sobre todo a partir de la investigación desarrollada por Pfeifer y Bongard (2007), quienes plantean que la diferencia fundamental en el sistema cognitivo humano se traduce en su relación con la periferia o mecanismos de aprehensión del entorno. En tal sentido, en el bucle carne y entorno-mundo es que se produce el proceso de comunicación, por lo tanto, debemos adentrarnos en la unidad de la percepción y el movimiento. En tal contexto, podemos situarnos en medida de inscribir en ello, al mismo tiempo, el proceso de conocer, partir desde ahí para comprender el lenguaje como fundamento del yo pienso: el lenguaje es al yo pienso lo que es el movimiento a la percepción. Por esto, "ningún Percibir no percibe a condición de ser Sí mismo de movimiento": esta fórmula no sólo significa que el sujeto que percibe debe ser por otra parte un sujeto motor, sino que la constitución de la cosa no puede ser cumplida sin referencia a las cinestesias.

Esta propuesta se sustenta en análisis consagrados a la sensación que desembocan sobre la idea de una "praktognosis", de un conocer y aprender desde y en la acción. En efecto, una vuelta por actuales investigaciones en visión (Palacios, 2005; Nôe, 2004, 2009; Thompson, 2007) permite evidenciar una significación motricia de cualidades sensibles, en particular del color, conseguido a través una actitud del cuerpo fenomenal antes que por una conciencia representativa: antes de ser un espectáculo objetivo, la cualidad se deja reconocer por un tipo de comportamiento que la pretende en su esencia y por eso en cuanto mi cuerpo adopta la actitud del azul obtengo una quasi-presencia del azul. Sin embargo, añade Merleau-Ponty (2000), la significación motricia de los colores solo se entiende si dejan de ser estados cerrados sobre ellos mismos o cualidades indescriptibles ofrecidas a la constatación de un sujeto pensante. Este bucle de la percepción y del movimiento posibilita la identidad de un ser y, por lo tanto, de su aprendizaje. De manera que al movimentar-se, explaya e imprime su conciencia, su conocimiento y su posibilidades futuras de conocimiento (Pfeifer y Bongard, 2007). En consecuencia, dentro del plano de la construcción de la virtualidad de su experiencia o de su acción, vale decir, de su lenguaje verbal, éste inevitablemente se genera desde la encarnación, desde el mundo de la percepción y movimiento (Lakoff y Johnson, 1999). De manera que el lenguaje siempre será una metáfora de la acción y de la experiencia en sí.

\section{TRANSICIÓN DE LA MOTRICIDAD (ACCIÓN) AL CONOCIMIENTO Y EDUCACIÓN}

Estos aspectos tienen una repercusión enorme en lo que intentamos construir como marco referencial y operativo de la presente investigación. Sobre todo desde el punto de vista de las posibilidades de estudios y de los campos de acción. Por ejemplo, si consideramos el primer punto, surge inmediatamente la pregunta: ¿qué conocemos acerca de los pormenores de la corporeidad y, por tanto, de la construcción del conocimiento en el 
contexto escolar? Al decir pormenores nos referimos a lo que la persona realiza día a día, como una serie de acciones que hasta el momentos han sido tratadas y contempladas como triviales e insignificantes o sólo desde un punto de vista morfo-funcional, tales como: comer, dormir, caminar, posiciones al sentarse y desplazarse, respirar, amplitud de acción y actitudes corpóreas, espacios relacionales y habitables, configuración geográfica, danza y juego, vestido y alimentación y su relación con la construcción del conocimiento, pero por sobre todo con el desarrollo humano. Específicamente, poco se ha profundizado, desde la Educación, en este sentido; cabe mencionar los trabajos realizados por Pozo (2001, 2003), Calvo (2005), Moreno (2005) y Grant (2007) sobre el clima, el ecosistema y la construcción social, entre otros aspectos.

Seguidamente, es menester señalar que la acción y, por tanto, el conocimiento, dentro de lo hemos llamado acoplamiento estructural (Varela, 1997) con el entorno, requiere de una característica y capacidad del ser humano que le permita, precisamente, entrar en un diálogo permanente con el mismo entorno, en el cual se encuentran otros seres como él-ella y distintos a él-ella. Dicha capacidad se reconoce como Empatía. Varela (2000) ha definido a la empatía como la emoción básica que ha permitido el desarrollo y evolución del ser humano como especie. Conjuntamente con él, Thompson $(2001,2007)$ ha realizado una categorización de la empatía. El tipo de empatía que nos interesa resaltar de acuerdo los intereses del proyecto, se caracteriza desde la dimensión encarnada de nuestra existencia, en el sentido que no es iniciada desde un proceso de pensamiento generado desde la persona misma, sino más bien que tal proceso se genera y operacionaliza en la vinculación o ligazón entre personas con similares características corpóreas cuyas bases experimentales e investigativas se centran en el trabajo de Rizzolatti (2001). En tal sentido, la empatía se genera en todo el actuar de la persona, en todo lo que se desprende y es observable como en todo aquello que internaliza y no es observable o por lo menos conscientizable desde el punto de vista de la expresión verbal o del análisis explícito. De manera que adquiere un estatus de percepción-acción. Varela y Thompson (2001) han expresado que tal fenómeno genera, desde el punto de vista de las neurociencias, una activación automática de nuestras propias áreas de la corteza cerebral acordes al comportamiento o acción que se está observando en otra persona, y con la cual se está produciendo un proceso de empatía. Los autores llaman a este tipo de empatía como un acoplamiento sensomotor. No obstante reconocen, como se señaló más arriba, que junto a este acoplamiento sensomotor se produce un acoplamiento afectivo o resonancia afectiva que consiste en un estado de mutua definición del estado emocional de cada uno.

En tal sentido, Damasio $(2001,2005)$ sostiene que el aprendizaje y en consecuencia la conciencia, se construye en la dinámica de los marcadores somáticos o emociones y la consecuente dislocación de la corporeidad. Es una cadena que no puede ser reducida a ningún de lo eslabones que la componen sino más bien sólo es comprensible en la configuración o fenómeno en sí. Por lo tanto lo que se aprende no es sólo aquello de lo que se trata la sesión, en el caso de la escuela, sino también y fundamentalmente, de cómo se trata la sesión. Vale decir, lo más próximo para el niño, dada esta capacidad biológica, es aprender de acuerdo a lo que el docente hace o la mayoría de sus compañeros hacen en tanto despliegue de la corporeidad del maestro y de sus compañeros. De manera que la acción y comportamiento del docente tiene mayor resonancia en el aprendizaje en el/a niño/a, de acuerdo al compromiso afectivo y activo (positivo o negativo) con el docente. Pues en él se reflejan, neuronalmente (Rizzolatti 2001, en Cosmelli 2005) la acción y el despliegue afectivo del profesor. 


\section{METODOLOGÍA}

\subsection{FASE CUANTITATIVA}

En función de establecer criterios de selección de casos sostenibles y concordantes con los objetivos de la investigación, se procedió a establecer un mecanismo de base metodológica complementaria, aunque de fondo el análisis fundamental se realiza desde procedimientos y paradigmas cualitativos.

En términos operativos se construyó una etapa cuantitativa previa que permitiera una descripción y agrupamiento global de los establecimientos educativos que superara la tradicional división por rendimiento y dependencia administrativa. Esto se describe a continuación.

\subsubsection{Variables construidas para análisis de conglomerados}

A fin de seleccionar casos para la fase cualitativa del estudio se agrupó los casos a través de un análisis de conglomerados, el que se realizó a partir de 5 variables que han sido definidas como relevantes para el estudio. Para esto se trabajó con microdatos del SIMCE 2008 para $8^{\circ}$ básico y 2007 para $4^{\circ}$ básico (solo se consideró establecimientos que tuvieran ambos cursos). Y encuesta a padres y apoderados que acompaña al SIMCE en los años y cursos respectivos. Luego de un arduo trabajo de limpieza y homologación de estas bases (ambas encuestas de padres y apoderados no tenían el mismo sistema de codificación, lo que obligó la recodificación de una de ellas), se consolidó una base única, la cual luego de excluir los casos que no presentaban puntaje o encuestas a padres y apoderados (al menos 15) en ambas mediciones del SIMCE, se encuentra constituida por 3954 casos.

En base a estos casos se construyeron las variables a incluir en el análisis de conglomerados. Estas son:

1. Puntaje SIMCE $4^{\circ}$ : Variable escalar construida como el promedio de los puntajes SIMCE de $4^{\circ}$ básico. Representa el nivel de conocimiento formal de los estudiantes de cuarto básico.

2. Puntaje SIMCE $8^{\circ}$ : Variable escalar construida como el promedio de los puntajes SIMCE de $8^{\circ}$ básico. Representa el nivel de conocimiento formal de los estudiantes de $8^{\circ}$ básico.

3. Situación socioeconómica: (Gráfico 1) Variable categorial operacionalizada como nivel de ingresos de las familias del establecimiento. Representa la clase social a la que pertenecen los estudiantes del establecimiento. A través de un análisis de conglomerado se distinguen cuatro grupos principales:

a) Establecimientos donde la mayor cantidad de hogares (94\%) tienen un ingreso inferior a 400.000, siendo especialmente relevante la cantidad de hogares $(71 \%)$ que declaran menos de 200.000 y menos de 100.000 (26\%).

b) Establecimientos en que el $90 \%$ presenta ingresos menores a 600.000 pero existe una baja presencia (menos del 6\%) de hogares con ingresos menores a 100.000 , concentrándose el $50 \%$ de las familias en el tramo 100.000 a 300.000.

c) Establecimientos en donde el ingreso de las familias es relativamente homogéneo entre 200.000 y 1.000 .000 concentrando en este tramo el $73 \%$ de los hogares.

d) Establecimientos en que sobre el $77 \%$ de los hogares declara ingresos sobre 1.000.000 y prácticamente no existen hogares con ingresos bajo $500.000(5 \%)$. 
Grafico 1. Distribución por ingreso familiar

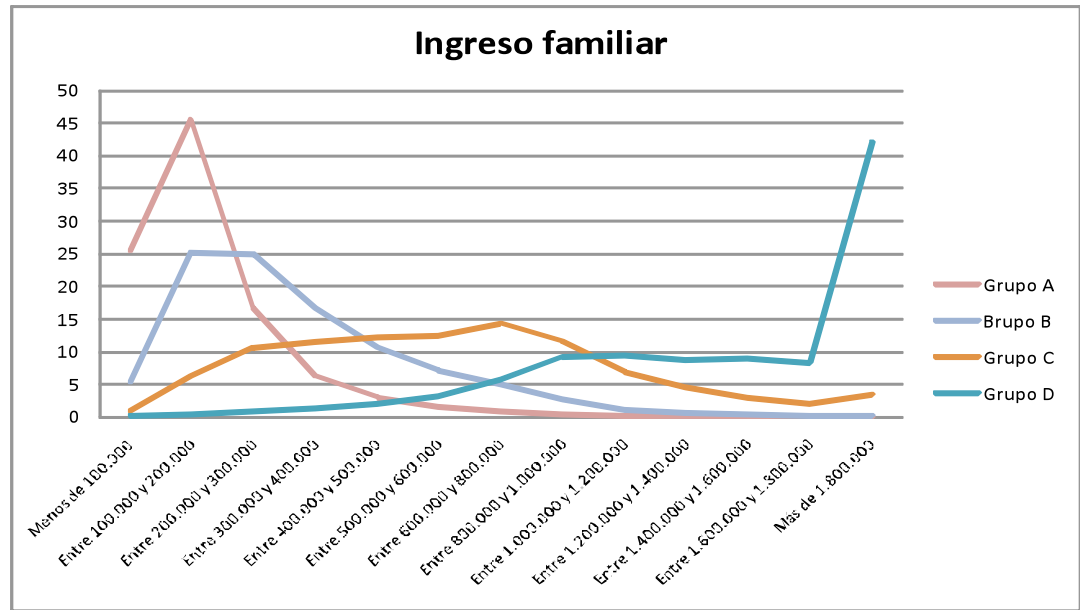

4. Nivel cultural: Variable categorial construida a partir del nivel educacional de ambos padres, cantidad de libros en la casa y origen étnico de ambos padres. Representa las características socioculturales del hogar de los hogares de los estudiantes del establecimiento. A través de un análisis de conglomerados se distinguen 4 grupos:

a) Establecimientos donde el porcentaje de pertenencia étnica tanto de los padres como de las madres si bien es relativamente bajo, es el más alto de la muestra $(9,9$ y 11,3 respectivamente). Son establecimientos donde la presencia de libros es bastante escasa, donde sobre el $40 \%$ declara tener menos de 5 libros en casa y el $87 \%$ menos de 30. Asimismo, la educación de ambos padres es la más baja de la muestra, presentando un alto porcentaje de padres y madres que no han culminado su enseñanza escolar (sobre $77 \%$ ) mientras que las/os que presentan educación superior, aunque sea incompleta, no supera el $4 \%$.

b) Establecimientos en que la pertenencia étnica es relativamente menor que en el primer grupo, aunque sigue existiendo un importante porcentaje, alrededor del $6 \%$ en los padres y $7 \%$ en madres. La presencia de libros es bastante más elevada que en el grupo anterior, aunque siguen existiendo cerca de un $22 \%$ de familias que declaran tener menos de 5 libros en casa y más de la mitad (56\%) declara tener más de 11 libros. Respecto al nivel educacional de los padres, en este grupo si bien cerca de la mitad de los padres no ha concluido la enseñanza escolar (54\% en padres y 55\% en madres), es notoria la presencia de padres que concluyen la educación escolar y que inclusive cursan educación superior $(10 \%)$.

c) Establecimientos en que la pertenencia étnica es bastante baja, la que no supera el $4 \%$ en ambos padres. La presencia de libros en el hogar es bastante más alta, donde menos del 10\% declara tener menos de 5 libros, declarando la mayoría $(51 \%)$ tener entre 6 y 30 libros en casa. La educación de los padres de este grupo es bastante alta, donde sobre el $36 \%$ posee estudios superiores y menos del $20 \%$ no ha completado su enseñanza escolar, tanto en padres como madres, presentando la mayor cantidad de casos una enseñanza media completa ( $42 \%$ en padres y $44 \%$ en madres). 
d) Establecimientos en que la pertenencia étnica es prácticamente nula (cercana al $1 \%)$, donde la tenencia de libros en el hogar es bastante alta (76\% declara tener sobre 30 libros) y la educación de ambos padres es alta, presentando sobre el $84 \%$ de los casos una educación superior, siendo prácticamente inexistentes los casos de no término de la enseñanza escolar (alrededor del 2\%)

5. Relacionalidad: Variable categorial construida en base a la aspiración educacional del estudiante, mecanismos de información de los padres y el parentesco con el estudiante del apoderado. Representa el tipo de relación que establecen los apoderados con la educación del estudiante. A través de un análisis de conglomerados se distingue 2 grupos:

a) Establecimientos donde las expectativas de estudios de los alumnos que tienen los apoderados se refieren principalmente a terminar la enseñanza media técnico profesional (37\%) y en menor medida continuar estudios superiores en Institutos Profesionales o Universidades (17 y 27\% respectivamente). Respecto a los mecanismos de información respecto a cuestiones relativas al establecimiento y el estudiante, la mayoría no maneja mayor información (44\%) y los que sí lo hacen es a través de las reuniones de apoderados (37\%). En este grupo, si bien la mayoría de los apoderados son los padres del estudiante, destaca que el $10 \%$ no son parientes y el $7 \%$ aprox. son otros parientes.

b) Establecimientos en que las expectativas de estudios se enfocan a que los estudiantes sigan estudios universitarios en pre y postgrado (79\%), siendo prácticamente inexistentes expectativas de salidas intermedias del sistema escolar. Respecto a los mecanismos de información, la mayoría no maneja información (34\%) mientras que los que sí están informados lo hacen a través de las reuniones de apoderados y medios de comunicación ( $31 \%$ y $16 \%$ respectivamente). En este grupo prácticamente todos los apoderados son los padres del estudiante $(93 \%)$.

\subsection{FASE CUALITATIVA}

Los resultados del estudio se presentan a partir del análisis de contenidos obtenidos mediante la observación participante en la cual se emplearon entrevistas semi estructuradas, registros de audio y video sobre la cotidianidad escolar y notas de campo.

De acuerdo a Pérez (1994), una investigación de carácter cualitativo puede plantearse en tres fases, como sigue:

a. Primera pre-activa: que consiste en clarificación de supuestos y fundamentación, focalización de la población, elección del procedimiento y la técnica, planeación de observaciones repetidas.

b. Segunda interactiva: implica un momento exploratorio que consiste en acercamientos procesuales a las realidades del colegio, naturalizar la presencia de la investigadora, interactuar. Otra instancia, de esta etapa, es el momento del mapeo en el que se configura un cuadro completo de los rasgos más relevantes de la situación o fenómeno objeto de análisis. La selección del tipo de situaciones, eventos, actores, lugares, momentos y temas que serán abordados en primera instancia en la investigación es decir el muestreo de espacios y escenarios, tiempos y momentos (Sandoval, 2002: 120).

La información diaria se registró mediante videos de situaciones, de audio y notas de campo. Posteriormente, se desarrollaron dos entrevistas grupales: una con profesores y profesoras, otra con estudiantes del grado octavo y dos entrevistas en profundidad con profesoras. 
c. Tercera pos-activa: Significa el procesamiento de información y análisis de la misma. La información sistematizada fue procesada mediante la técnica análisis de contenido que de acuerdo con Abela (2003) consiste en interpretación de textos, ya sean escritos, grabados, pintados, filmados u otra forma diferente mediante la cual se abren las puertas al conocimiento de diversos aspectos y fenómenos de la vida social. Se empleó como soporte para la categorización de patrones y relaciones el programa Atlas-ti.

\subsubsection{Descripción de los casos de estudio}

La selección de los casos responde a criterios definidos en el marco producido por el análisis de Cluster mencionado anteriormente. A partir de dicho análisis, se procedió a seleccionar desde los seis grupos de establecimientos un caso de estudio para cada uno, observando como criterio fundamental la accesibilidad y disposición del mismo para con la investigación. Los cursos escogidos, al interior de cada caso, obedecieron fundamentalmente de ambos ciclos del nivel, fundamentalmente, en los terminales de cada ciclo $\left(4^{\circ}\right.$ y $\left.8^{\circ}\right)$.

\subsubsection{Unidad de análisis}

Relaciones cotidianas de acuerdo a interpretaciones de las personas participantes en función del rol, el sexo y la etapa del ciclo vital actores, según se muestra en la siguiente tabla:

Tabla 1. Unidad de Análisis

\begin{tabular}{|c|c|c|}
\hline Unidad de análisis & Universo/Participantes & Muestra \\
\hline $\begin{array}{l}\text { Interacción desde } \\
\text { la corporeidad en el } \\
\text { contexto escolar. }\end{array}$ & $\begin{array}{l}\text { Interacciones en contextos } \\
\text { escolares, niveles: } \\
\text { Lugares: salas de clases, patios de } \\
\text { recreo, baños, pasillos, oficinas de } \\
\text { administración, comedor, gimnasio. } \\
\text { Roles: personal administrativo, } \\
\text { cuerpo docente, estudiantes. } \\
\text { Ciclo vital: personas niños, } \\
\text { púberes (adolescentes en algunos } \\
\text { casos), personas jóvenes, personas } \\
\text { adulto(as). }\end{array}$ & $\begin{array}{l}\text { Momentos, lugares y } \\
\text { personas significativas } \\
\text { para los y las } \\
\text { estudiantes y profesores } \\
\text { - profesoras del nivel } \\
\text { básico. }\left(4^{\circ} \text { y } 8^{\circ}\right)\end{array}$ \\
\hline
\end{tabular}

La categoría transversal sobre la cual navega el análisis es la corporeidad que, de acuerdo con la fundamentación teórica, engloba los sentires, los pensamientos, las acciones que configuran algunas de los entramados relacionales que se dan en la cotidianidad.

\subsubsection{Técnicas e instrumentos de recolección de datos}

Atendiendo al carácter de la investigación las técnicas e instrumentos de recolección de datos tienen diferentes gradientes de especificidad, que se presentan de manera introductoria en la tabla 2: 
Tabla 2. Técnica e instrumentos

\begin{tabular}{|c|c|}
\hline Método & Investigación cualitativa en campo \\
\hline Técnica & Observación participante \\
\hline Instrumentos & $\begin{array}{c}\text { Registros y notas de campo, documentos } \\
\text { audiovisuales, entrevistas individuales y } \\
\text { grupales a estudiantes y profesores (as) }\end{array}$ \\
\hline
\end{tabular}

\subsubsection{Analisis y discusión}

De acuerdo a los resultados productos de los diferentes intrumentos de recogida de datos, se pudo estructurar una matriz de relaciones que permita sintetizarlos de manera más compresibe y manipulable. En este sentido, se consideró lo expresado por los informantes claves en función de sus sentires y lógicas de explicitación de sus comprensiones frente a lo preguntado. De tal manera, la contrucción de una tabla de doble entrada permite explicitar los procesos globales que se configuran desde la dimensión corporea y su visualización externa diferenciada desde el relato de los propios actores. Este proceso se reiteró en cada caso a fin de dar confirmabilidad y credibilidad, tanto a las categorías diseñadas como a sus indicadores a partir de los informantes claves de los seis casos estudiados.

La matriz que recoge categorías de expresión evidenciados en tres escenarios de relacionalidad, a saber: las clases tipo A son llamadas a las que los y las estudiantes les atribuyeron características agradables y que les motivaban; las denominadas clases tipo B son las que no cuentan con valoraciones positivas mayoritariamente; y los espacios de recreo y encuentro que refiere a las vivencias alrededor de este espacio-tiempo.

Tabla 3. Categorías resultantes

\begin{tabular}{|c|c|c|c|c|}
\hline Categorías & Sub categorías & $\begin{array}{c}\text { Clases } \\
\text { tipo A }\end{array}$ & $\begin{array}{c}\text { Clases } \\
\text { tipo B }\end{array}$ & $\begin{array}{c}\text { Espacios } \\
\text { de recreo y } \\
\text { encuentro }\end{array}$ \\
\hline \multirow{2}{*}{ Miradas } & Frente & $\mathrm{X}$ & & $\mathrm{X}$ \\
\cline { 2 - 5 } & Abajo & & $\mathrm{X}$ & $\mathrm{X}$ \\
\hline \multirow{2}{*}{ Posturas } & Abiertas - disposición & $\mathrm{X}$ & & $\mathrm{X}$ \\
\cline { 2 - 5 } & Cerradas- contención & & $\mathrm{X}$ & \\
\hline \multirow{2}{*}{ Orientación } & Frente & $\mathrm{X}$ & & $\mathrm{X}$ \\
\cline { 2 - 5 } & Lado & $\mathrm{X}$ & $\mathrm{X}$ & $\mathrm{X}$ \\
\cline { 2 - 5 } & Espaldas & & $\mathrm{X}$ & \\
\hline \multirow{2}{*}{ Gestos } & Agrado & $\mathrm{X}$ & & $\mathrm{X}$ \\
\cline { 2 - 5 } & Desagrado & & $\mathrm{X}$ & \\
\hline \multirow{2}{*}{$\begin{array}{c}\text { Contactos } \\
\text { directo }\end{array}$} & Presentes & $\mathrm{X}$ & & $\mathrm{X}$ \\
\cline { 2 - 5 } & Ausentes & & $\mathrm{X}$ & \\
\hline
\end{tabular}




\begin{tabular}{|c|c|c|c|c|}
\hline \multirow{2}{*}{ Despliegues } & Activos & $\mathrm{X}$ & & $\mathrm{X}$ \\
\cline { 2 - 5 } & Reactivos & & $\mathrm{X}$ & \\
\hline \multirow{2}{*}{ Proxemia } & Variable & $\mathrm{X}$ & & $\mathrm{X}$ \\
\cline { 2 - 5 } & Estática & & $\mathrm{X}$ & \\
\hline \multirow{2}{*}{$\begin{array}{c}\text { Control } \\
\text { fisiológico }\end{array}$} & autónomo & $\mathrm{X}$ & & $\mathrm{X}$ \\
\cline { 2 - 5 } & heterónomo & & $\mathrm{X}$ & \\
\hline Lenguaje & Fund. analógico & $\mathrm{X}$ & & $\mathrm{X}$ \\
\cline { 2 - 5 } & Fund. digital & & $\mathrm{X}$ & $\mathrm{X}$ \\
\hline
\end{tabular}

\section{APRENDIZAJE Y CONOCIMIENTO ESCOLAR. HALLAZGOS}

Como hemos revisado, Pozo (2001) propone la importancia de acentuar que los procesos de construcción de conocimiento o aprendizaje de los mismos se constituyen desde las relaciones y desde el involucramiento del estudiante a partir de sus dinámicas senso-motroras en los mencionados aprendizajes, vale decir, aquello que se realiza desde el cómo, fundamentalmente, más que del qué. Incluso autores como Neus Sanmarti (2003, 2009), desde la didáctica de las ciencias experimentales, sostiene que sería mas pertinente referirnos al conocimiento escolar más que al aprendizaje de contenidos, sobre todo en virtud de reconocer que el conocimiento al fin y al cabo se traducen en determinados patrones de comportamiento e interpretación del mundo, desde esos mismos patrones de acción. De lo contrario no pasan de ser considerados como procedimientos burocráticos e intrascendentes dentro de la vida del estudiante o escolar.

Los resultados descritos en la Tabla $\mathrm{N}^{\circ} 3$ presentan que las clases tipo A son aquellas que involucran una participación de los estudiantes que posibilita el mayor compromiso de recursos corpóreos que en el plano de la cognición; se traduce en un estar y actuar en el mundo desde la emocionalidad, favoreciendo los procesos relacionales tanto con el contenido y de esta forma, convirtiéndolo en significado (Bruner, 2006) de una forma más fluida al decir de los informantes claves... "cuando la clase es mas entretenida, todo es más bacán, como que es más fácil entender a la profe...la materia y el tiempo pasa más rápido, como que no te dai cuenta que estai en clases" (UH2).

En tal sentido, los despliegues de cada estudiante como también su capacidad de ensayar una y otra vez sus posibilidades decisionales en el mismo proceso, constituye un factor sustantivo en el momento de la comprensión de sus propios procesos de acuerdo a los pretextos de relación que se actualizan en las sesiones de clases. Del mismo modo, en el sentido contario, la pasividad y poca actividad, sumado a un distanciamiento emocional, provoca los efectos de evasión y resistencia frente a la situación. Lo que conduce, por consiguiente, hacia procesos de aprendizaje favorecedores de la simulación o del intento de hacer creer que se está aprendiendo lo instruido por el docente. En palabras de otro informante clave: "muchas veces las clases son tan aburridas, que es difícil mantenerse atento, por eso prefiero hacer como que estoy escuchando a la profe, pero en realidad, estoy haciendo otra cosa" (UH2). Agrega: "Yo sé lo que cada profe quiere y trato de dar eso, aunque no sé para que me sirve a mí, pero así puedo pasar el curso y tener buenas notas"(UH2). 
De manera que el/a escolar se sitúa en una posición fundamentalmente estratégica en función de la relación de poder que el/a docente tiene para decidir su aprobación o no dentro del curso o materia en desarrollo, a diferencia de la sesión que resulta agradable, en las cuales el contenido y la atmósfera se funden en una sola experiencia.

Desde ese mismo punto de análisis, Howard (2004) y Candela (2006) enfatizan la importancia de las dinámicas interaccionales entre los docentes y los estudiantes, en la enseñanza escolar, en virtud de establecer los patrones de acción y de contingencia que se generan y que al fin y al cabo constituyen lo que definimos como aprendizaje, mas allá de los objetos o contenidos de las mismas interacciones. Esto no quiere decir que el contenido en tanto información u objeto de la relación no tenga sentido en sí mismo, más bien se trata de puntualizar que el mismo contenido se despliega o vitaliza para bien o para mal desde y en las acciones que se ejercen. Por lo tanto, si sólo se trata de hablar de un contenido u objeto de enseñanza lo que fundamentalmente se aprende en la niñez, siguiendo tanto a Piaget (2001), Vayer (1999) como a Vigostky (1999), serían las formas de ese hablar más que el fondo de ese hablar. De manera que el conocimiento escolar es aquello que se aprende en virtud de las interacciones y relaciones recurrentes en planos de emocionalidad confluyentes e interpretables por un espacio simbólico común, en un determinado patrón de contingencias tanto normativas, espaciales como institucionales que definen y condicionan el actuar concreto de los niños y niñas, que puede ser explicitado en las categorías arriba señaladas.

A partir de los estudios realizados por el autor (Toro, 2005, 2007, 2010a, 2010b), se puede señalar que básicamente esta situación no difiere en lo que respecta a otros tipos de contenido formales, toda vez que no se aprecia con claridad qué es lo que aprenden los niños o cuál es su conocimiento desde sus acciones, sino más bien se sabe lo que no aprenden si consideramos los resultados de las pruebas nacionales tales como el SIMCE y la PSU. Por tanto, es una nebulosa saber qué aprenden los niños y niñas dada la escases de investigaciones que describen ni menos comprenden dicho fenómeno. Sin embargo, y a partir de aquello que se observó y registró, la sesión misma de la clase, las dinámicas relacionales definida como espacio de interacción y las acciones propiamente tal, emprendidas por los escolares de $4^{\circ}$ y $8^{\circ}$ básico, se orientan a la reproducción o imitación de modelos pre-establecidos y a negociar los tiempos de la clase, a simular aprendizajes en función de la aprobación. Dado que la mayoría de las clases se orientan hacia el Tipo B. Tal situación puede llevarnos a inferir que lo aprendido, como acción, es la repetición y la negociación en primer lugar, pero el contenido formal, propiamente tal, queda en suspenso o a criterio del docente.

En este mismo sentido, se ha podido establecer, que es el/la docente quien define los tiempos, normas y patrones de acción, no sólo desde el discurso y uso del poder, sino, sobre todo, desde las dinámicas disposicionales y de acción de acuerdo al contexto de los contenidos desarrollados, los planos de emoción permitidos, los tiempos de organización personal y social, las formas de distribución espacio-temporal y, por consecuencia, de los aprendizajes buscados. Tal aspecto nos lleva con naturalidad a visualizar que los procesos formales son de dependencia absoluta de los docentes; por tanto, genera una relación sustentada en el control externo del tiempo de "trabajo serio" que, en definitiva, es el tiempo que tiene valor en los procesos de evaluación formal.

A diferencia, en los tiempos de recreo y encuentro, por cortos o largos que ellos sean, las decisiones son tomadas o por los líderes o por acuerdos entre niños y niñas, 
pero dichas acciones rara vez son consideradas dentro del espacio formal; por el contrario, responden a criterios completamente diferenciados y obviamente reconocidos de esa manera por los/as escolares. Los recreos en general demandan presencia de adultos que, más que participar de las dinámicas recreacionales de los niños y niñas, cumplen la tarea de cuidar, vigilar o controlar las mismas. Por tanto, lo que ocurre en dichos espacios, en términos de vínculo y reconocimiento en los espacios de "trabajo serio", no tiene lugar.

\section{LA ESCUELA "DE VERDAD"}

"Para mí...el colegio comienza, de verdad después de almuerzo, en los talleres...pues ahí puedo realizar mis sueños...jugar fútbol.”

"Para mí también, es el tiempo donde estamos en lo que de verdad queremos...en mi caso es bailar” (UH3).

Las dinámicas relacionales que se generan en las tardes dentro de los que podemos comprender como espacio-tiempos que responden a diferentes realidades y demandas (Jornada Escolar Completa, Actividades de Libre Elección), dependiendo del tipo de administración del establecimiento, responde obviamente con particularidades, pero es un espacio que tiende a ser reconocido como el lugar de mayor participación o menor grado de control adulto.

Consecuentemente, la escuela como espacio de relaciones se genera por lo menos en dos niveles o momentos, el espacio formal, fundamentalmente adulto y de instrucción, y en el espacio de la tarde que es menos adulto y mas de encuentro en función de los interes que los niños/as podrían manifestar dependiendo de las posibilidades y proyecto educativo del establecimiento. Aspecto que no es menor dado que, en general, la oferta es reducida y también obedece a criterios de selección explícita o solapada. De igual forma, la situación del recreo y las relaciones que allí ocurren no tienen lugar como contenido formal dentro del espacio de "trabajo serio", sino más bien como un espacio anexo y que tiene un valor compensatorio y, en algunos casos, publicitario, dependiendo de los resultados que se tengan en determinados eventos de convocatoria local, regional o nacional de algunas disciplinas-actividades que se realicen en los establecimientos. Se destaca, no obstante, que representar al colegio tiene un valor relacional diferenciado entre los informantes, dado que tal situación generalmente es reconocida en casos de éxito y la participación por sí sola no sería valorada realmente, pues no tiene un producto que mostrar para dar cuenta de la educación del establecimiento.

Por otra parte, y sobre la base de que la cognición emerge de la capacidad de volver sobre la acción emprendida, de la recurrencia y recursividad de la misma (Pfeifer y Bongard, 2007), es comprensible entender que en los casos estudiados se presenta dinámicas diferenciadas en función de desarrollar lógicas de dependencia y heteronomía en los diversos niveles de actuación de los actores involucrados. Es decir, la escasez de la toma de decisiones explícitas, el obedecer, el permanecer quieto, el control fisiológico externo, son elementos recurrentes y recursivos dentro de la dinámica de los casos estudiados, posibilitando de esta forma que los procesos de pensamientos se generen desde esa base vivencial y relacional. Lo cual no potencia la experiencia permanente de tomar decisiones autónoma, desde los mismos procesos señalados, salvo en los casos de mantención y búsqueda de un beneficio posterior (aprobar, por lo tanto, debo simular) y de 
subordinación. Obviamente, dicha situación no es uniforme entre los casos, pero difieren precisamente en la intensidad de esta situación, como en la plataforma sociocultural que genera alternativas de actuación interna y externa al establecimiento.

En consecuencia, no sería desventurado levantar la hipótesis de que las diferencias se producen desde las acciones que los alumnos emprenden en la cotidianidad de su vida escolar, lo que empuja o posibilita aprendizajes, los cuales pueden ser pertinentes o no a los orientados desde los proyectos educativos particulares y al currículo oficial.

Finalmente, podemos señalar que la identificación de ciertas categorías conceptuales que permiten avanzar hacia la caracterización y comprensión del aprendizaje, específicamente desde la corporeidad, tales como: configuración corpórea o imagen corporal, la proxémica, lo espacio-temporal y su disposición tanto en la persona como los demás dentro de su contexto de acción, los procesos de interacción que se construye y despliega desde lo corpóreo, constituyen elementos que permiten explicitar de manera más pertinente los aprendizajes o, dicho de otra manera, qué de los enseñado se aprende, en definitiva, desde la vivencia y acción-inter-acción del/a estudiante (Gallagher 2009, 2010; Pfeifer y Bongard, 2007, Moreno 2005).

\section{COROLARIOS}

En relación a todo el trabajo investigativo desarrollado, se puede establecer dos tipos de aspectos a considerar como consecuencias del mismo:

a. Consecuencias desde los procedimientos cuantitativos: Si bien no fue parte sustantiva de esta investigación el establecer un análisis descriptivo estadístico de los casos estudiados, cabe al menos señalar que los resultados de análisis de conglomerados, en función de las variables construidas, resulta a lo menos preocupante. Pues se puede visualizar que, al interior del sistema educativo chileno, se presentan verdaderos guetos o grupos bastante diferenciados unos de otros y que no es descartable realizar ciertas predicciones a partir de la pertenencia a uno u otro grupo. En particular, lo que se refiere al ingreso familiar (ver Gráfico 1) y el nivel cultural de la familia. Elemento sustantivo en el momento de establecer las diferencias tanto de dinámicas relacionales favorecidas entre los estudiantes entre sí, como con los docentes y el contenido de enseñanza.

b. En relación a los procesos relacionales: en este aspecto, por lo demás sustantivo de la investigación, nos encontramos con procesos ambivalentes en la escuela, centrados en procesos de resistencia u oposición desde la expresión como comprensión formulada por los informantes claves. En tal sentido las dinámicas relacionales se sustentan en aspectos burocráticos y formalismos que, en definitiva, decantan en aprendizaje cercanos a mantenerse dentro del sistema.

Tal consideración nos replantea el aprendizaje orientado desde el docente, como un proceso tanto aislado como lineal en relación Estimulo-Respuesta, propias de la tradiciones conductivas, muy renegadas pero muy usadas en el escenario escolar y social. De manera que, en los casos estudiados, el aprendizaje se torna un proceso paralelo al contenido, incluso puede hasta ser opuesto.

Más bien, deberíamos acordar en que "El aprendizaje del animal no es sobre un estímulo externo particular, sino que es aprendizaje sobre una situación como totalidad. Es un cambio contextual complejo lo que produce la modificación sobre los patrones... el 
conocimiento del animal según su historia evolutiva es intencionalidad" (Freeman, 2007: 46). Por consiguiente, los aprendizajes que inevitablemente se producen en la relación y dinámica escolar podrán ser más o menos asertivos en la medida que asumimos la cultura y las condiciones de habitabilidad, acción y construcción de ciudadanía. Procesos que, hasta el momento, se producen, en general y de acuerdo a los casos chilenos estudiados, al margen y en contra de la sala de clase, pues en ella se aprende a depender y a obedecer, a contener y replicar, pero no a crear y construir.

Por lo tanto, en el caso de una memorización de una determinada información, lo que se aprendería sería la acción de memorizar, no la información en sí. Esto sería más claro en niños y niñas en enseñanza escolar básica, toda vez que se encuentran en procesos de desarrollo neurológico sensible (Fernández, 2003). Por consiguiente, si aceptamos que los procesos de pensamiento tienen su emergencia en determinadas formas de acción y actuación de la dimensionalidad corpórea de cada ser humano que se encuentre siempre en comunicación, y si observamos, una vez más, los datos del SIMCE y de la PSU, aparte de mantenerse las brechas históricas de acuerdo a la dependencia administrativa y la condición socio-económica del último año -asumiendo que la dependencia administrativa no sólo implica un carácter formal sino también un contexto de interacción, de cultura y de hacer-, pareciera estar claro que los aprendizajes se construyen y son pertinentes a las dinámicas relacionales encarnadas y situadas en las posibilidades de comunicación y desenvolvimiento de los/as alumnas en sí mismos.

\section{REFERENCIAS BIBLIOGRÁFICAS}

Bruner, J. (2006). Los actos de significado. Más allá de la revolución cognitiva. Barcelona: Nueva Alianza.

Candela, A. (2006). "Construcción discursiva y la ciencia en el aula". Quintanilla, M.; Aduriz, A. (Eds)., Diálogos didácticos. Santiago: Editorial UC.

Calvo, C. (2005). "Entre la educación corporal caótica y la escolarización corporal ordenada". Revista Iberoamericana de Educación, n. 39, 91-106.

Damasio, A. (2000). Sentir lo que sucede. Cuerpo y emoción en la fábrica de la consciencia. Santiago: Andrés Bello.

Damasio, A. (2005). En busca de Spinoza. Neurobiología de la emoción y los sentimientos. Barcelona: Crítica.

Davinson, R.; Jackson, D. y Kalin, N. (2000). Emotion, plasticity, context and regulation. Psychological Bolletin, vol. 126, n. 2, 890-909.

Gallagher, S. (2007). Introduction: The arts and sciences of the situaded body. Janus Head, vol. 9, n. 2, 293-295.

Gallagher, S. y Zahavi (2009). The phenomenological mind. London: Routledge.

Gallagher, S. y Schmicking, D. Edit. (2010). Handbook of phenomenology and cognitive science. New York: Springer.

Fernández, V. (2003). Neurociencias y aprendizaje. Santiago: UCSH.

Freeman, W. (2007). "Dinamicas no lineales e intencionalidad: el rol de las teorías cerebrales en las ciencias de la mente". Ibañez, A. y Cosmelli, D. (Comp.), Nuevos enfoques de la cognición - Redescubriendo la dinámica de la acción, la intención y la Intersubjetividad. Santiago: Universidad Diego Portales.

Lakoff, G. y Johnson, M. (1999). Philosophy in the flesh, the embodied mind an its challenges in the western thougth. New York: Basic Books. 
Llinas, R. (2003). El cerebro y el mito del yo. Bogotá: Norma

Maturana, H.; Varela, F. (1998). De máquinas a seres vivos. Santiago: Dolmen.

Merleau-Ponty, M. (2000). La fenomenología de la percepción. Barcelona: Peninsula.

Nöe, A. (2004). Action in perception. Massachusetts. MIT Press.

Nöe, A. (2009). Out of our heads. Why you are not your brain, and other lessons from the biology of consciousness. New York: Hill \& Wang.

Pfeifer, R. y Bongard, J. (2007). How the body shapes the way we think. A new view of intelligence. Massachusetts: MIT Press.

Sergio, M. (2002). Da educaçao física a motricidade humana. Madeira: Universidade de Madeira.

Solms, M. y Turnbull, O. (2004). El cerebro y el mundo interior. Bogotá: Fondo de Cultura Económica.

Thompson, E. (2002). Emphaty and consciuosness. Journal of Consciuosness Studies, n. 8, 5-7, 1-32.

Thompson, E. (2007). Mind and life. Biology, phenomenology, and the sciences of mind. Massachusetts: Belknap Harvard.

Toro, S. (2005). "Motricidad y mente encarnada". Libro de actas IV Congreso Internacional de Motricidad Humana. Porto do Son.

Toro, S. (2007). "Aproximación epistemológica a la didáctica de la motricidad desde el discursos y práctica docente, conclusiones y proyecciones". Estudios Pedagógicos, vol. XXXIII, n. 1, 45-57.

Toro, S. (2010a). Lenguaje y motricidad: la acción visible como texto y expresión. Cinta de Moebio, n. 37. En línea; disponible en www.moebio.uchile.cl

Toro, S. (2010b). "Neurociencias y aprendizaje. Un texto en construcción”. Estudios Pedagógicos vol. XXXVI, n. 2, 323-341.

Valsiner, J. (2000). Culture and Human development. New York: Sage.

Varela, F.; Thompson, E.; Rosch, E. (1997). De cuerpo presente. Las ciencias cognitivas y la experiencia humana. Barcelona: Gedisa.

Varela, F. y Thompson, E. (2001). Radical embodiment: Neural dynamics and consciuosness. Trends in Cognitive Sciences, vol. 5, n. 10, 418-425.

Varela, F. (2000). El fenómeno de la Vida. Santiago: Dolmen. 